\title{
Airway Delivery of Low-Dose Miglustat Normalizes Nasal Potential Difference in F508del Cystic Fibrosis Mice
}

\author{
Bob Lubamba ${ }^{1}$, Jean Lebacq ${ }^{2}$, Patrick Lebecque ${ }^{4}$, Rita $_{\text {Vanbever }}^{3}$, Anissa Leonard ${ }^{4}$, Pierre Wallemacq ${ }^{1}$, \\ and Teresinha Leal ${ }^{1}$
}

Departments of ${ }^{1}$ Clinical Chemistry, ${ }^{2}$ Cell Physiology, ${ }^{3}$ Pharmaceutical Technology, and ${ }^{4}$ Pediatric Pulmonology, Université Catholique de Louvain, Brussels, Belgium

\begin{abstract}
Rationale: N-butyldeoxynojyrimicin (NB-DNJ, miglustat [Zavesca]) an approved drug for treating Gaucher disease, was reported to be able to correct the defective trafficking of the F508del-CFTR protein. Objectives: To evaluate the efficacy of in vivo airway delivery of miglustat for restoring ion transport in cystic fibrosis (CF).

Methods: We used nasal transepithelial potential difference (PD) as a measure of sodium and chloride transport. The effect of nasal instillation of a single dose of miglustat was investigated in F508del, cftr knockout and normal homozygous mice. The galactose iminosugar analog $\mathrm{N}$-butyldeoxygalactonojirimycin (NB-DGJ) was used as a placebo.

Measurements and Main Results: In F508del mice, sodium conductance (evaluated by basal hyperpolarization) and chloride conductance (evaluated by perfusing the nasal mucosa with chloride-free solution in the presence of amiloride and forskolin) were normalized 1 hour after an intranasal dose of 50 picomoles of miglustat. Chloride conductance in the presence of $200 \mu \mathrm{M}$ 4-4' -diisothiocyanostilbene2,2'-disulphonic acid (DIDS), an inhibitor of alternative chloride channels, was much higher after miglustat than after placebo. In cftr knockout mice, a normalizing effect was observed on sodium but not on chloride conductance.

Conclusions: Our results provide clear evidence that nasal delivery of miglustat, at picomolar doses, normalizes sodium and Cftr-dependent chloride transport in F508del transgenic mice; they highlight the potential of topical miglustat as a therapy for CF.
\end{abstract}

Keywords: cystic fibrosis; miglustat; chloride transport; sodium transport; nasal potential difference

Cystic fibrosis (CF) is caused by mutations of the cystic fibrosis transmembrane conductance regulator (CFTR) gene resulting in malfunctioning or reduction of the CFTR protein through a variety of mechanisms $(1,2)$. The protein has an essential role in transepithelial ion transport, functioning as an ohmic, small conductance, cAMP-dependent chloride channel but also regulating many other transport proteins, most notably the epithelial sodium channel $(\mathrm{ENaC})(3,4)$. The most prevalent $\mathrm{CF}$-causing mutation, F508del, is a misfolded protein that fails to escape the endoplasmic reticulum (ER) and reach the apical membrane of many epithelial cell types (5-7).

The loss of CFTR function can be assessed in vivo by measuring the transepithelial nasal potential difference (PD), which has been increasingly used as an index of therapeutic efficacy in novel fundamental therapies either in patients $(8,9)$ or in animal models (10).

Despite the clear link between abnormal ion transport and $\mathrm{CF}$, the pathogenesis of lung disease in CF is complex and still de-

(Received in original form January 12, 2009; accepted in final form March 17, 2009)

Correspondence and requests for reprints should be addressed to Teresinha Leal, M.D., Ph.D., Department of Clinical Chemistry, St. Luc University Hospital, $10 \mathrm{Av}$ Hippocrate, B-1200 Brussels, Belgium. E-mail: teresinha.leal@uclouvain.be

Am J Respir Crit Care Med Vol 179. pp 1022-1028, 2009

Originally Published in Press as DOI: 10.1164/rccm.200901-00490C on March 19, 2009 Internet address: www.atsjournals.org

\section{AT A GLANCE COMMENTARY}

Scientific Knowledge on the Subject

Miglustat, an approved oral therapy for Gaucher disease, has been shown to partially correct defective trafficking of F508del-CFTR.

\section{What This Study Adds to the Field}

Nasal deposition of miglustat improves both chloride and sodium transport in F508del-CFTR mice, warranting further studies of topical miglustat as potential therapy for cystic fibrosis.

bated. The initiating event in CF-airway disease pathogenesis seems to be reduced airway surface liquid volume that develops, in large part, as a consequence of abnormal ion transport. A compound capable of rescuing the F508del protein function and of normalizing ion transport abnormalities could be considered, in contrast to symptomatic or alleviating treatments currently available, as a disease-modifying therapeutic agent and would be beneficial for most patients with $\mathrm{CF}$.

The generation of animal models that mimic the human phenotype has been an important achievement in CF research. To date, 13 mouse models have been created and 3 F508del strains are available (11). The prevalence of the F508del mutation along with its severe phenotype makes the F508del-CF mouse a useful model to test novel pharmaceutics that target the mutant CFTR processing and turnover (10).

Miglustat, an N-alkylated iminosugar (n-butyldeoxynojyrimicin, [NB-DNJ], Zavesca) is an orally available drug approved for use in patients with nonneuronopathic, type I Gaucher disease, an inherited glycosphingolipid lysosomal storage disorder (12-14). The drug has also been considered in the treatment of other glycosphingolipidoses $(15)$ as well as viral infections $(16,17)$. Its efficacy has been investigated in CF experimental models such as F508del mutant airway epithelial cells in culture or isolated tissues from mice (18-22). Oral treatment with miglustat in patients with $\mathrm{CF}$ is now being evaluated within a pilot phase $2 \mathrm{a}$ clinical trial at our CF center (http://clinicaltrials.gov/ct2/show/ NCT00742092). Miglustat is an inhibitor of several enzymes, including the ceramide-specific glucosyltransferase, disaccharidases, and $\alpha$-glucosidases. Inhibition of the former enzyme, which initiates the glycosphingolipid biosynthetic pathway and catalyzes the formation of glucocerebroside, provides the rationale for the use of miglustat in glycophospholipid storage diseases (12-15). Inhibition of intestinal disaccharidases, such as sucrase, maltase, and lactase, has been related to the high incidence of diarrhea, flatulence, and abdominal pain in patients orally treated with miglustat. Inhibition of $\alpha$-glucosidase has been suggested as the underlying mechanism of the CFTR corrector effect of miglustat in CF tissues (18-20). The corrective effect of miglustat 
has been identified in CF cell cultures grown on impermeable plastic dishes, a configuration that allows interaction of drugs through the apical side of epithelia (18-22). Therefore, we hypothesized that in vivo topical application of miglustat to the airway epithelium is able to restore transepithelial ion transport abnormalities of the F508del-CFTR protein. In the present work, we investigated in CF mice homozygous for the F508del mutation (23), in cftr knockout mice, and in their corresponding wild-type homozygous normal mice, the effect of in vivo treatment by nasal instillation of miglustat on parameters of the transepithelial potential difference (PD) across the nasal mucosa. Our results provide clear evidence that local delivery of miglustat, at picomolar doses, to the airway epithelium counteracts sodium transport even in the absence of CFTR, and activates CFTR-dependent chloride transport in F508del-CF mice. These data highlight the potential of topical miglustat as a therapy for $\mathrm{CF}$.

\section{METHODS}

\section{Animal Model}

The study investigated CF mice homozygous for the F508del-CFTR mutation in the $129 /$ FVB outbred background ( $\left.c f t r^{\text {tm1 Eur }}\right)(23)$ and their wild-type normal homozygous littermates, which were housed at the Animal Care Facility of our University following recommendations of the Federation of European Laboratory Animal Science Associations (24). Mice were 3 to 4 months of age and weighed between 20 and 30 grams. To prevent CF intestinal obstruction, Movicol $(55.24 \mathrm{~g} / \mathrm{L}$; Norgine, Heverlee, Belgium) was administered in acidified drinking water. The mice were genotyped at 21 days of age by using Taqman quantitative polymerase chain reaction (PCR), as previously described (25). C57Bl/6 $c f t r^{\mathrm{UNC}}$ knockout mice and their wild-type controls were also investigated. All experiments were approved by the local Ethics Committee for Animal Welfare and conformed to the European Community regulations for animal use in research $\left(\right.$ CEE $\left.N^{\circ} 86 / 609\right)$.

\section{Pharmacological Agents}

Miglustat was applied by nasal instillation of $5 \mu$ l solution by using a pipette (26). $\mathrm{N}$ - butyldeoxygalactonojirimycin (NB-DGJ), a galactose iminosugar analog without $\alpha$-glucosidase-inhibitory activity (27), was used as a placebo at $100 \mu \mathrm{M}$ concentration. In dose-response experiments, a nasal PD test was performed after nasal deposition, in each nostril, of saline or miglustat solution applied at different concentrations varying from $0.1 \mu \mathrm{M}$ to $100 \mu \mathrm{M}$. Topical nasal application of iminosugars was performed under light sedation induced over 5 minutes after intraperitoneal injection of $0.25 \mathrm{mg}$ midazolam (Dormicum; Roche, Brussels, Belgium). To allow uniform dispersal of intranasal deposited drug, both nostrils were subsequently instillated with the same volume of solution and the animal was kept for 2 to 3 minutes in an upright position to prevent an unknown amount of drug being coughed up or swallowed. The effect on nasal PD parameters was examined 1 hour or 6 hours after miglustat administration. Iminosugar drugs were purchased from Toronto Research Chemicals (Toronto, ON, Canada). All other chemicals were from Sigma Aldrich (St. Louis, MO). Solutions with iminosugars were prepared in saline, stored at $4^{\circ} \mathrm{C}$, and used within 4 days after preparation.

\section{Nasal Potential Difference Measurements}

Nasal PD measurements were performed under general anesthesia as previously described $(10,28)$. In brief, a double lumen catheter was placed in a nasal passage, and a needle inserted in the subcutaneous space in a hind leg served as a reference bridge. Solutions were perfused at a constant rate of $12 \mu \mathrm{l} / \mathrm{min}$. The following parameters were recorded: (1) the most negative stable baseline PD value (PDmax); (2) decrease in PDmax after perfusion with basal solution containing $10^{-4} \mathrm{M}$ amiloride; (3) chloride secretion as subsequent cumulative changes in PD after perfusion with zero chloride solution containing amiloride and with $10^{-5} \mathrm{M}$ forskolin (SumCl); (4) change in response to addition of $200 \mu \mathrm{M}$ 4,4'-diisothiocyanostilbene-2,2'-disulphonic acid (DIDS), an alternative chloride channel inhibitor. The composition of basal isotonic saline solution was (mM): $\mathrm{Na}^{+} 140, \mathrm{Cl}^{-} 120, \mathrm{~K}^{+} 5.2, \mathrm{HCO}_{3}{ }^{-} 25, \mathrm{HPO}_{4}{ }^{-} 2.4$, $\mathrm{H}_{2} \mathrm{PO}_{4}^{-} 0.4, \mathrm{Ca}^{+2} 1.2, \mathrm{Mg}^{+2} 1.2, \mathrm{pH}$ 7.4. Chloride was replaced isoosmotically by gluconate in the chloride-free solution. The small junction potential resulting from replacement from basal solutions to zero chloride solutions was measured (maximum to $1.4 \mathrm{mV}$ ) and used to correct PD values recorded with zero chloride solution.

\section{Statistics}

Descriptive statistics (mean and SEM) and tests of significance were performed using SAS-JMP software (SAS Institute, Cary, NC). Betweengroup comparisons were evaluated using one-way analysis of variance. Posthoc comparisons were made using Student's $t$ test or Tukey-Kramer test for two or more $x$ levels, respectively. Null hypothesis was rejected at $P<0.05$.

\section{RESULTS}

As illustrated in representative tracings (Figure 1), F508del animals showed typical ion transport abnormalities similar to those observed in patients with CF (29-31); increased PDmax and amiloride response, and reduced chloride secretion. Indeed, mean values of PDmax were significantly different between F508del mice and their corresponding wild-type normal homozygous littermates (Figure 2A). Mean SumCl voltage values were reduced by more than half in F508del animals compared with the corresponding values measured in control mice (Figure 2B).

\section{Nasal Deposition of Miglustat Reduces Sodium Transport in F508del Mice}

Nasal deposition of miglustat and NB-DGJ was well tolerated and no adverse effect was observed. A nasal PD test was
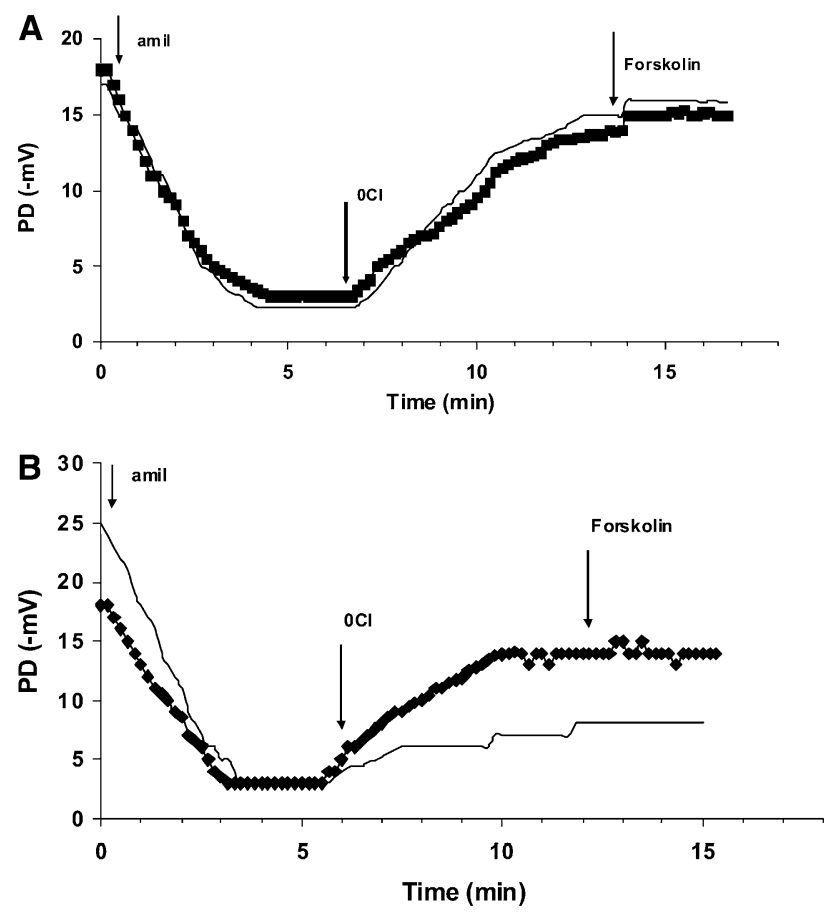

Figure 1. Representative tracings of nasal transepithelial potential difference (PD) measurements in (A) wild-type and (B) F508del mice 1 hour after a single nasal instillation in each nostril of $5 \mu \mathrm{l}$ of $100 \mu \mathrm{M}$ miglustat or placebo (NB-DG]). Tracings show sequential response of the nasal mucosa to perfusion consecutively with basal solution, basal solution containing $10^{-4} \mathrm{M}$ amiloride (amil), chloride-free solution plus amiloride $(0 \mathrm{Cl})$, and chloride-free solution with amiloride plus $10^{-5} \mathrm{M}$ forskolin (forskolin). Arrows indicate change of solutions. Smooth line $=$ placebo; square line = miglustat. 
A
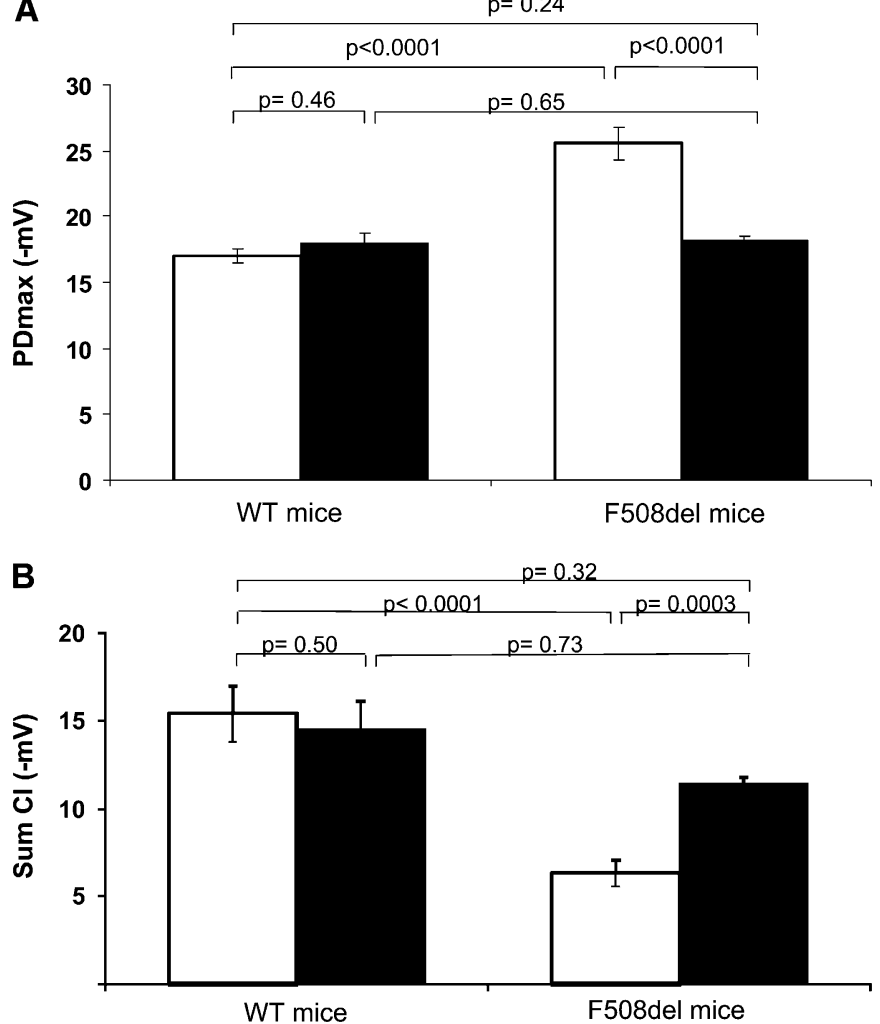

Figure 2. (A) Maximal baseline potential difference (PD) values (PDmax) and (B) stimulated chloride transport (SumCl) in wild-type (WT) homozygous control and F508del mice 1 hour after a single intranasal deposition in each nostril of $5 \mu \mathrm{l}$ of $100 \mu \mathrm{M}$ miglustat or placebo (NB-DGJ). Chloride transport was evaluated by the cumulative changes in nasal PD after perfusion with chloride-free solution in the presence of $10^{-4} \mathrm{M}$ amiloride and $10^{-5} \mathrm{M}$ forskolin. Bars represent means \pm SEM for 5 to 7 animals per group. Open bars = placebo; solid bars $=$ miglustat. $P$ values denote levels of significance measured in miglustat-treated group compared with the corresponding placebotreated group or to the wild-type group.

undertaken in F508del and wild-type mice 1 hour after nasal instillation with miglustat or placebo. The concentration of $100 \mu \mathrm{M}$ was initially selected because correction of ion transport was reported in epithelial cells in culture 2 hours after incubation in the presence of this concentration in the culture medium (18). Placebo experiments were performed 1 hour after nasal instillation of $5 \mu \mathrm{l}$ of $100 \mu \mathrm{M}$ of the galactose iminosugar analog, NB-DGJ (27).

As illustrated in Figures 1A-1B, the tracing profile obtained in F508del mice 1 hour after nasal instillation of miglustat was similar to that observed in placebo-treated wild-type mice. A significant reducing effect on PDmax was detected in F508del mice while comparing miglustat-treated to placebo-treated animals (Figure 2A). PDmax in F508del mice after miglustat reached identical values to those recorded in placebo wild-type mice (Figure 2A). The normalizing effect of miglustat on sodium conductance in F508del animals was confirmed on the amiloride sensitivity. Indeed, mean $( \pm$ SEM $)$ values of amiloride response were higher in placebo-treated $(22.1 \pm 1.2 \mathrm{mV} ; \mathrm{n}=7)$ than in miglustat-treated F508del mice $(15.8 \pm 1.5 \mathrm{mV} ; \mathrm{n}=5 ; P=0.005)$. No significant effect of miglustat was detected in the normal homozygous control group, neither on basal PD values (Figure 2A) nor on amiloride response (data not shown).
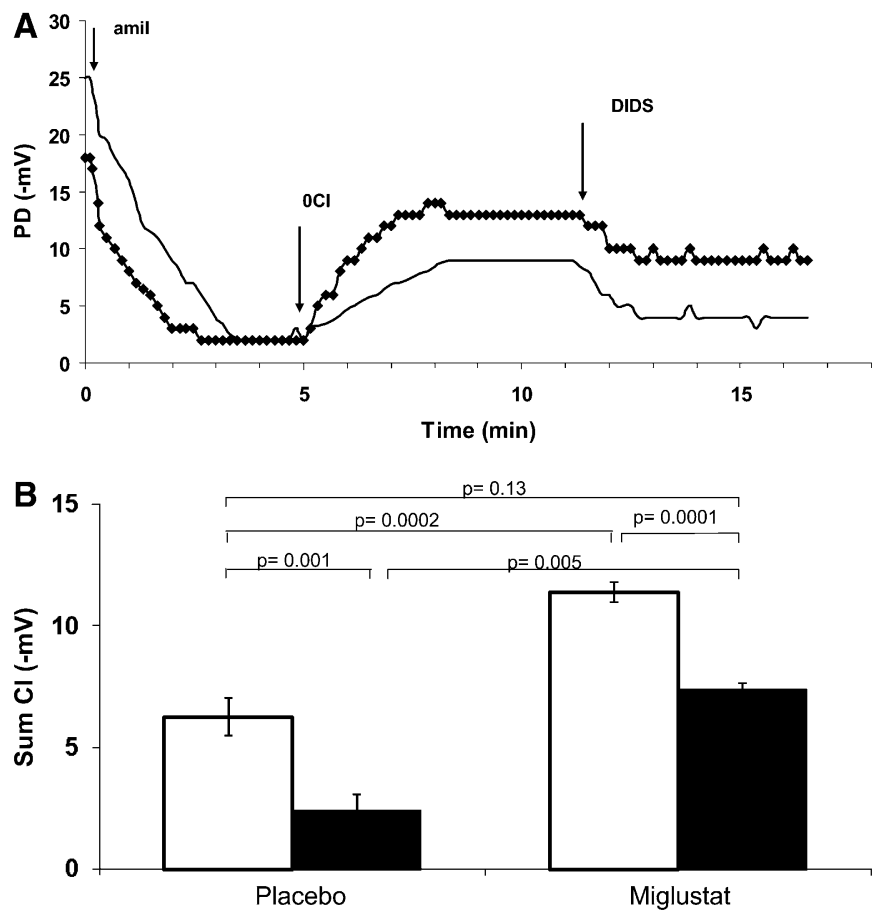

Figure 3. Nasal transepithelial potential difference (PD) measurements in F508del mice 1 hour after nasal deposition of $5 \mu$ of $100 \mu \mathrm{M}$ miglustat or placebo (NB-DG]) in the presence or in the absence of $4,4^{\prime}$-diisothiocyanostilbene-2-2'-disulphonic acid (DIDS). (A) Representative tracings show sequential response of the nasal mucosa to perfusion consecutively with basal solution, basal solution containing $10^{-4} \mathrm{M}$ amiloride (amil), chloride-free solution plus amiloride $(\mathrm{OCl})$ and chloride-free solution containing amiloride and $200 \mu \mathrm{M}$ DIDS. Smooth line = placebo; square line $=$ miglustat. Arrows indicate changes of solutions. (B) Chloride secretion evaluated by perfusion of the nasal mucosa with chloride-free solution plus $10^{-4} \mathrm{M}$ amiloride in the presence or in the absence of $200 \mu \mathrm{M}$ DIDS. Bars represent means \pm SEM for 5 to 7 animals per group in each group. Open bars $=-$ DIDS; solid bars $=+$ DIDS. $P$ values denote levels of significance measured in DIDS-treated group compared with the corresponding non-DIDS-treated group or placebo-treated group.

\section{Nasal Deposition of Miglustat Increases Chloride Transport in F508del Mice}

A significant effect on chloride transport was detected in F508del mice 1 hour after nasal deposition of $5 \mu \mathrm{l}$ in each nostril of $100 \mu \mathrm{M}$ miglustat (Figure 1A-1B). Indeed, chloride conductance was twofold higher in F508del mice treated with miglustat compared with the corresponding placebo-treated group (Figure 2B). Moreover, SumCl values in F508del mice after miglustat treatment were comparable to those values recorded in placebotreated wild-type group (Figure 2B). No significant effect was observed in the wild-type group when comparing miglustattreated to placebo-treated animals (Figure 2B).

\section{Influence of Alternative Chloride Transport Pathways on the Effect of Miglustat}

To determine whether the effect of nasal instillation of miglustat in increasing chloride transport across the nasal epithelium of F508del mice is dependent on alternative chloride transport pathways, we examined the effect of the drug and of its ion transport inactive analog, NB-DGJ, in the presence of DIDS, a non-CFTR dependent inhibitor of chloride transport. Representative PD tracings obtained in F508del mice 1 hour after administration of 

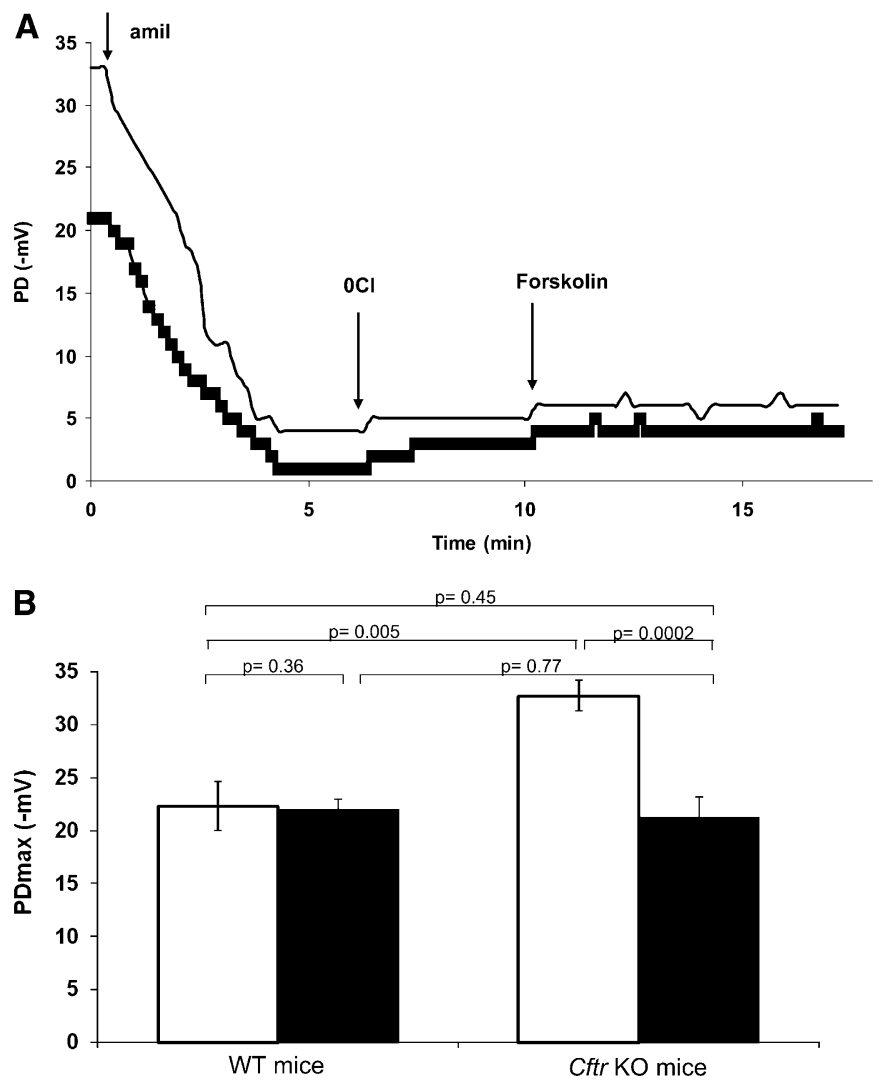

Figure 4. Nasal transepithelial potential difference (PD) measurements in cftr knockout (KO) mice and corresponding wild-type (WT) mice 1 hour after intranasal deposition of $5 \mu \mathrm{l}$ of $100 \mu \mathrm{M}$ miglustat or placebo (NB-DGJ). (A) Representative tracings show sequential response of the nasal mucosa to perfusion consecutively with basal solution, basal solution containing $10^{-4} \mathrm{M}$ amiloride (amil), chloride-free solution plus amiloride $(\mathrm{OCl})$ and chloride-free solution containing amiloride and $10^{-5} \mathrm{M}$ forskolin. Smooth line = placebo; square line = miglustat. Arrows indicate changes of solutions. (B) Maximal baseline PD values (PDmax). Bars represent means \pm SEM for four animals per group in each group. Open bars = placebo; solid bars = miglustat. $P$ values denote levels of significance measured in miglustat-treated group compared with the corresponding NB-DGJ-treated group or to the WT group.

$5 \mu \mathrm{l}$ of $100 \mu \mathrm{M}$ miglustat or placebo, and following addition of $200 \mu \mathrm{M}$ DIDS diluted in chloride-free solution containing amiloride and forskolin are depicted in Figure 3A. As we have previously shown (10), alternative chloride transport pathway contributes to the largest part of the residual chloride conductance in the nasal mucosa of F508del mice. Indeed, perfusion of the nasal mucosa of placebo-treated F508del mice with DIDS reduces chloride conductance to a very low level (Figure 3A-3B). However, in the miglustat-treated group, PD changes after DIDS remained at least threefold higher than those values recorded in the placebo-treated group $(P=0.005$; Figure $3 \mathrm{~B})$. DIDS-sensitive chloride transport was of the same magnitude in placebotreated $(-3.9 \pm 0.5 \mathrm{mV})$ as in miglustat-treated F508del mice $(-4.0 \pm 0.4 \mathrm{mV} ; P=0.82)$. These findings indicate that the effect of miglustat in F508del-CF mice does not principally depend on alternative chloride transport pathway.

\section{Nasal Deposition of Miglustat on Ion Transport in Cftr Knockout Mice}

To determine whether the effect of miglustat on ion transport is dependent on the presence of CFTR protein, a nasal PD test was

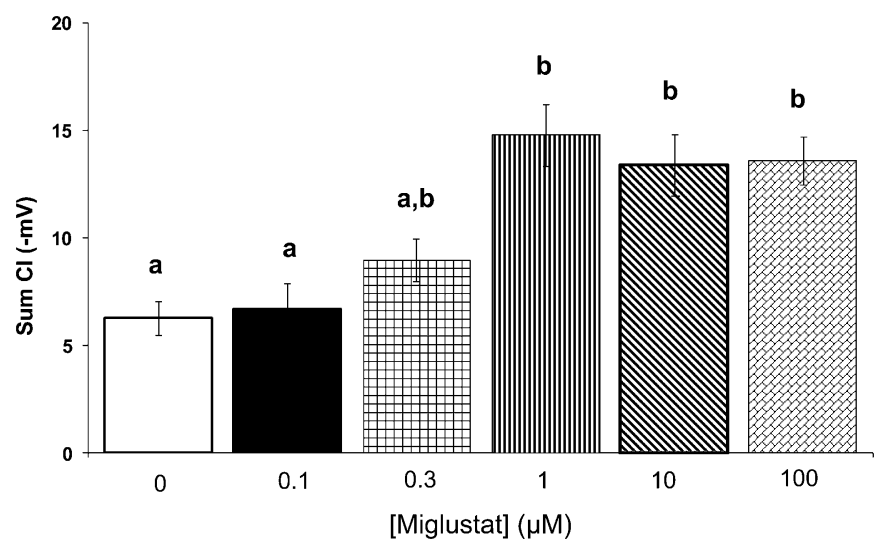

Figure 5. Dose-dependent effect of chloride transport function by nasal instillation of miglustat in F508del mice. A nasal transepithelial potential difference (PD) test was performed 1 hour after intranasal deposition of $5 \mu \mathrm{l}$ in each nostril of saline or miglustat solution varying from 0.1 to $100 \mu \mathrm{M}$. Chloride transport was evaluated by the cumulative changes in nasal PD after perfusion with chloride-free solution in the presence of $10^{-4} \mathrm{M}$ amiloride and $10^{-5} \mathrm{M}$ forskolin. Bars represent means $\pm \mathrm{SEM}$ for five mice in each group. Levels not connected by same letter are significantly different at a two-sided $\alpha$ level of 0.05 .

undertaken in $\mathrm{C} 57 \mathrm{Bl} / 6 \mathrm{cftr}^{\mathrm{UNC}}$ knockout animals 1 hour after nasal deposition of $5 \mu \mathrm{l}$ of $100 \mu \mathrm{M}$ miglustat or placebo and in the corresponding wild-type mouse groups. As illustrated in Figure 4A, cftr knockout mice also demonstrated a characteristic ion transport profile consistent with increased sodium and reduced chloride conductance. After intranasal miglustat, a normalizing effect was observed on sodium but not on chloride conductance (Figure 4A). Indeed, mean PDmax values were significantly reduced in miglustat-treated as compared with placebo-treated $c f t r$ knockout mice (Figure 4B). Mean chloride conductance values remained unchanged $(-2.7 \pm 1.3 \mathrm{mV}$ in placebo-treated vs. $-1.8 \pm 0.6 \mathrm{mV}$ in miglustat-treated knockout animals; $P=$ $0.52)$.

\section{Dose-dependent Effect of Nasal Instillation of Miglustat on Ion Transport in F580del Mice}

To investigate the dose-dependent effect of the intranasal deposition of miglustat in normalizing ion transport in $\mathrm{CF}$, a nasal PD test was performed in F508del mice 1 hour after nasal instillation of saline or the drug applied at different doses: $5 \mu \mathrm{l}$ in each nostril of solution of a concentration varying from 0.1 to $100 \mu \mathrm{M}$ (i.e., a dose varying from 0.5 to $500 \mathrm{pmol}$ in each nostril). After nasal deposition of $10 \mu \mathrm{M}$ miglustat, PDmax values $(-18.2 \pm$ $1.0 \mathrm{mV} ; \mathrm{n}=5)$ were similar $(P=0.75)$ to those recorded after $100 \mu \mathrm{M}$ miglustat (Figure 2A). No effect was observed on sodium conductance following treatment with $1 \mu \mathrm{M}$ miglustat. Indeed, mean PDmax values after $1 \mu \mathrm{M}$ miglustat $(-25.4 \pm 1.2 \mathrm{mV})$ were similar $(P=0.75)$ to the corresponding values obtained in nontreated F508del animals $(-23.2 \pm 2.1)$. The subthreshold dose on sodium conductance in F508del mice was confirmed for all lower doses tested (data not shown). These data indicate that the $10 \mu \mathrm{M}$ solution corresponded to the lowest monitored dose of miglustat capable of normalizing the sodium conductance in the presence of mutant CFTR protein.

The $1 \mu \mathrm{M}$ intranasal solution corresponded to the lowest monitored dose giving a normalizing effect on chloride conductance. As illustrated in Figure 5, after $1 \mu \mathrm{M}$ intranasal miglustat, $\mathrm{SumCl}$ voltage changes were of the same magnitude as the values recorded after deposition of $100 \mu \mathrm{M}$ miglustat, a similar maximal normalizing effect being observed with the $10 \mu \mathrm{M}$ solution. A 


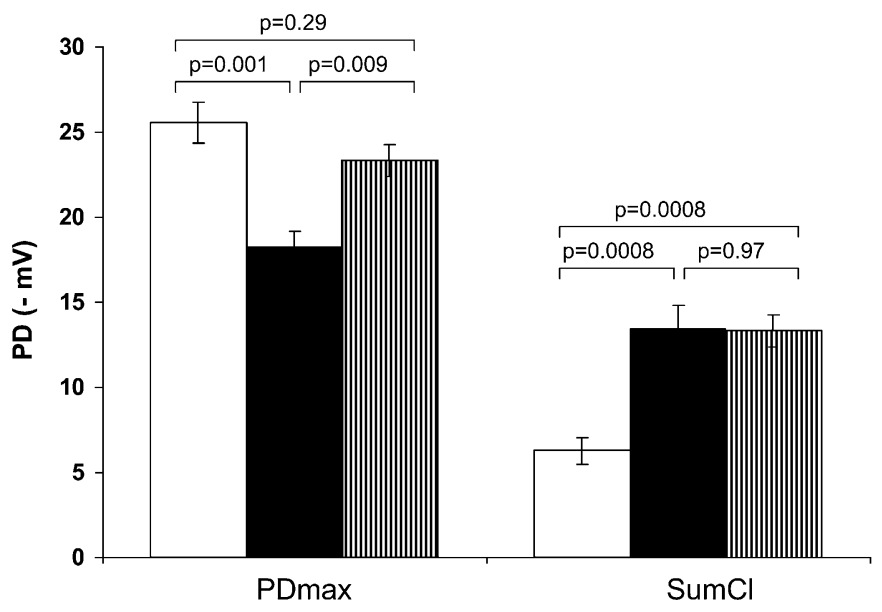

Figure 6. Duration of the effect of intranasal miglustat on sodium and chloride conductance in F508del mice. Maximal baseline potential difference (PD) values (PDmax) and stimulated chloride transport (SumCl) 1 hour or 6 hours after a single intranasal deposition of $10 \mu \mathrm{M}$ miglustat or placebo (NB-DG) in F508del mice. Chloride transport was evaluated by the cumulative changes in nasal PD after perfusion with chloride-free solution in the presence of $10^{-4} \mathrm{M}$ amiloride and $10^{-5} \mathrm{M}$ forskolin. Bars represent means \pm SEM for five to seven animals per group. Open bars = placebo; solid bars $=1$ hour miglustat; striped bars $=6$ hours after miglustat. $P$ values denote levels of significance measured in each group of cystic fibrosis (CF) mice compared with the placebo group.

subthreshold concentration on chloride transport was found to be $0.1 \mu \mathrm{M}$ miglustat, as no significant difference was observed between chloride conductance recorded after treatment with this solution and no treatment (Figure 5). An intermediate effect on chloride conductance was obtained with nasal delivery of the $0.3 \mu \mathrm{M}$ solution (Figure 5). These data indicate that a 10 -fold lower concentration, compared with that able to normalize sodium conductance, is required to restore CFTR-dependent chloride transport. These data also show that $5 \mu \mathrm{l}$ of $10 \mu \mathrm{M}$ miglustat, corresponding to $50 \mathrm{pmol}$, is the optimal lowest intranasal dose able to normalize both sodium and chloride transepithelial transport in F508del CF mice.

\section{Duration of the Effect of Nasal Instillation of Miglustat on Ion Transport in F580del Mice}

Profiles of the nasal PD were next undertaken in F508del mice 1 hour or 6 hours after intranasal instillation of the $10 \mu \mathrm{M}$ solution, which was selected as the optimal lowest intranasal dose. Under these conditions, no effect was observed on sodium conductance. Indeed, although it was normalized 1 hour after deposition of $10 \mu \mathrm{M}$ miglustat, 6 hours after treatment the basal hyperpolarization in F508del animals no longer differed from that recorded in the placebo-treated group (Figure 6). The normalizing effect on chloride conductance, however, persisted at the same value as that recorded 1 hour after treatment (Figure 6). These data indicate that the normalizing effect of picomolar intranasal dose of miglustat on chloride conductance persisted for at least 6 hours, whereas the duration of sodium transport normalization was shorter.

\section{DISCUSSION}

The present work was designed to test the hypothesis that topical delivery of miglustat into the nasal mucosa is able to overcome the ion transport defects of the mutant F508del-CFTR protein. A potential beneficial effect of miglustat in CF has been suggested through experimental studies performed in CF cell-culture lines and excised animal tissues (18-22). It has been reported that 2 hours exposure of $100 \mu \mathrm{M}$ miglustat to F508del-CFTR expressing cell-culture lines promotes an increase by $12 \%$ of the expression of mature CFTR protein to the apical cell membrane; this finding was associated with activation by $55 \%$ of the transepithelial chloride transport (18). The finding that, in different experimental protocols (18-22), miglustat has been added to polarized cell cultures grown on impermeable plastic dishes, an epithelial configuration allowing interaction of drugs through its apical side, provided the rationale for investigating the efficacy of in vivo deposition of miglustat in the airways.

A proof-of-concept phase 2 a clinical trial based on oral treatment of miglustat is being conducted in patients with $\mathrm{CF}$ in our center. Apart from transient tremor or exacerbation of tremor reported in approximately $20 \%$ of patients on treatment with miglustat, diarrhea and weight loss are common side effects occurring in about 85 and $60 \%$ of treated patients, respectively (12-15). Whereas osmotic diarrhea appears to be the result of the disaccharidase inhibitory activity, it is unclear whether weight loss results from the diarrhea and associated gastrointestinal symptoms such as abdominal pain and flatulence, a decrease in food intake, or a combination of these or other factors. Because of the high incidence of clinically important adverse reactions (12-15), studies using CF animal models may be of relevance for evaluating the potential therapeutic efficacy of the delivery of miglustat directly to the airways before innovative development programs that target miglustat as an inhaling medication for $\mathrm{CF}$ can be started and before randomized, controlled trials in patients with CF can be designed. The F508del mouse model used in this work (23) shows low but detectable residual CFTR activity, nearly normal mRNA expression levels of the mutant CFTR protein, and high survival rates when compared with the two other F508del mouse models $(32,33)$. Reduced chloride and increased sodium transport capacity across the respiratory epithelium are found in the F508del mouse model we use. Taken together, these properties make this model a useful in vivo system for studying novel therapeutic strategies that aim to activate chloride and reduce sodium transport function.

The preferred route of administration of any drug is one that gives direct access to its target. The choice of route is clearly influenced by the anatomical target site affected by the disease and by the physicochemical, pharmacological, and toxicological properties of the drug or vehicle used. Topical administration of drugs along the airway tree through the inhalation of a nebulized liquid or dry powder formulation has been used extensively in the treatment of $\mathrm{CF}$ and other pulmonary diseases. The large airway surface area and its decreased metabolic capacity relative to the gastrointestinal tract offer an attractive portal for airway delivery of drugs. The physiochemical properties of miglustat as a low molecular weight and a highly water-soluble molecule eliminating the need to use potentially toxic solvents would represent additional advantages. Small aerodynamic size particles formed, for instance, by nebulization of an aqueous solution could efficiently deliver miglustat to the lower lungs for a local effect (34). Deposition of miglustat in the airways would advantageously minimize systemic undesirable effects and the amount of drug required to locally achieve therapeutic levels.

In the present work, we showed that in vivo deposition of a picomolar dose of miglustat applied directly to the respiratory epithelium efficiently corrects ion transport abnormalities of the F508del-CFTR protein. The response was characterized by an early and complete correction of both sodium and chloride conductances. When administered orally (20), correcting effects of miglustat on the F508del mouse nasal mucosa required a dose 
approximately 120 -fold larger than the human therapeutic dose used in Gaucher disease $(1,200 \mathrm{mg} / \mathrm{kg} / \mathrm{d}$ to mice vs. $\sim 10 \mathrm{mg} / \mathrm{kg} / \mathrm{d}$ or $200 \mathrm{mg}$ three times a day to adult patients). The duration of the normalizing effect on chloride conductance could be related to the effective half-life of miglustat, reported to be 6.3 hours in humans (12). This is in keeping with a report that the correcting effect of the miglustat to CF cells in culture is time dependent; the effect of a final concentration of $100 \mu \mathrm{M}$ progressively declined after 4 to 6 hours with a total loss of effect after 14 hours (22).

The precise mechanism of action of miglustat for correcting chloride and sodium transport of F508del-CFTR protein is not clear. Our data showed that chloride transport after perfusion with DIDS, a commonly used inhibitor of alternative chloride channels (35) and electrogenic anion exchangers (36) in murine epithelia, was much higher in F508del mice in the presence of miglustat when compared with the galactose iminosugar derivative. This observation, in addition to the finding that the effect on chloride conductance was lost in cftr knockout mice, supports the notion that the normalizing effect of miglustat on chloride transport involves F508del-CFTR and not an alternative chloride transport pathway. It has been shown that miglustat prevents the interaction of F508del-CFTR protein to the chaperone calnexin (18), an ER-localized lectin-like protein involved in the CFTR biogenesis. Effectively, calnexin binds N-linked glycans, and two such glycosylation sites have been identified on CFTR in the extracellular loop four of the membrane spanning domain (MSD)-2 (37). Knockdown of calnexin leads a large portion of nascent CFTR to misfold $(37,38)$. Yet, the mechanism for calnexin action in folding and quality control of CFTR is not well understood, and studies on the role of calnexin in degradation and rescue of misfolded F508del-CFTR protein have provided mixed results. Although the overexpression of calnexin seems to favor accumulation of F508del-CFTR in the ER (38), siRNA-mediated down-regulation of calnexin does not inhibit degradation of wild-type or mutant F508del-CFTR $(37,38)$. Investigation has shown that binding to calnexin is not essential for degradation of wild-type or misfolded F508del-CFTR. Folding defects of the mutant protein have been evidenced either before or after the calnexin-dependent association of MSD-1 and MSD-2 (39). Therefore, the inhibition of ER $\alpha$-1,2-glucosylation of nascent CFTR protein by miglustat is probably not sufficient to completely restore the localization and the functional rescue of the misfolded protein as suggested (18). It could be possible that miglustat interferes with the efficiency level of the quality control of CFTR maturation and that normalizing drug effects could be associated with a less efficient quality-control system as observed in our F508del-CF mouse model that shows low but detectable residual CFTR function.

Our data are in agreement with those from Noel and colleagues (20) in which sodium conductance in CF airway epithelial cultured cells and excised nasal mucosa from F508del mice were normalized after miglustat treatment. Data from this group (20) have shown that the magnitude of the amiloride-sensitive current is similar in the presence or the absence of forkolin/genistein, intended to open CFTR channels, arguing that activation of F508delCFTR is not required for the down-regulating effect of miglustat on sodium conductance. However, as no significant effect on amiloride-sensitive current could be observed in nasal mucosa excised from orally treated $c f t r$ knockout mice (20), the authors concluded that the effect of miglustat is due to F508del-cftr rescue. In contrast, data from our work indicate that the corrective effect of miglustat on sodium conductance does not seem to be dependent on the functional rescue of CFTR. Indeed, a normalizing effect of intranasal deposition of up to $10 \mu \mathrm{M}$ miglustat solution on sodium conductance was clearly evidenced in the absence of cftr protein. Moreover, in the presence of the mutant protein, the duration of the effect was shorter, and a 10-fold higher effective dose was required for normalizing sodium conductance compared with the dose able to restore chloride conductance. A highly variable dataset with large dispersion around the mean (20) might, at least partly, explain differences between the work from Noel and colleagues (20) and ours. Likewise, the choice of the route of administration and the distinct dose potencies required for normalizing chloride and sodium conductances could additionally contribute to the differences found. As the positive effect of miglustat on chloride secretion remained evident in the absence of normalization of sodium transport, it does not support a counteracting effect of ENaC on CFTR. This is in keeping with studies performed in the $\beta \mathrm{ENaC}$ overexpressing mouse model (40) that develops a severe CF-like lung disease with airway liquid depletion, mucus retention, neutrophilic infiltration, but not the other aspects of CFTR dysfunction (41). Further preclinical pharmacokinetic and NPD in vivo studies assessing different doses and routes of administration, including airway delivery, to be conducted in the $\beta E N a C$ mouse model (40), would help confirm the finding that miglustat acts on sodium conductance through a direct effect on ENaC activity.

Another interesting observation of the effect of miglustat in $\mathrm{CF}$ is that, apart from its normalizing effect on ion transport, the drug has an antiinflammatory action (21). Actually, it has been reported that exposure for 24 hours to $200 \mu \mathrm{M}$ of miglustat (but also to its ion transport inactive analog NB-DGJ) reduced the expression of proinflammatory mediators induced by Pseudomonas aeruginosa infection, tumor necrosis factor (TNF)- $\alpha$, or IL-1 $\beta$ in bronchial epithelial cells in culture. Considering that airway inflammation is a hallmark of CF lung disease, further confirmation of the antiinflammatory effect of miglustat on topical delivery of picomolar doses to the airway tree could be largely beneficial for $\mathrm{CF}$ airway disease.

Besides reducing cellular levels of glycosphingolipids, miglustat, as an inhibitor of glucosylceramide-based glycosphingolipids, conversely induces ceramide accumulation (27). Recent studies have shown that both patients with CF and cftr knockout mice display lower levels of ceramide in plasma, lungs, and other CFaffected organs (42). The regulation of ceramide levels seems to be crucial for the maintenance of proper membrane permeabilization properties and homeostasis of inflammatory responses. It could therefore be postulated that, as evidenced for fenretinide, a semisynthetic retinoid (42), beneficial effects of miglustat might, at least partly, be mediated by correction of the ceramide defect.

We demonstrate that local delivery of a single picomolar dose of miglustat, an approved drug for oral treatment of Gaucher disease, to the airways of F508del-CF mice corrects both reduced chloride and exaggerated sodium transport. The effect is apparently dependent on CFTR-mediated chloride transport. Our data provide a strong rationale for airway delivery of miglustat for patients with CF lung disease.

Conflict of Interest Statement: None of the authors has a financial relationship with a commercial entity that has an interest in the subject of this manuscript.

Acknowledgment: Cftrtm1EUR (F508del (FVB/129) breeding pairs were obtained from Erasmus MC Rotterdam (b.scholte@erasmusmc.nl) with support from the EEC EUROCARECF program (EU FP6 LSHM-CT-2005-018932). The authors thank the French CF Association, Vaincre la Mucoviscidose, for providing Cftr knockout mice.

\section{References}

1. Rowe SM, Miller S, Sorscher EJ. Cystic fibrosis. N Engl J Med 2005;352: 1992-2001.

2. Davis PB. Cystic fibrosis since 1938. Am J Respir Crit Care Med 2006; 173:475-482.

3. Stutts MJ, Rossier BC, Boucher RC. Cystic fibrosis transmembrane conductance regulator inverts protein kinase A-mediated regulation 
of epithelial sodium channel single channel kinetics. J Biol Chem 1997;272:14037-14040.

4. Ismailov II, Awayda MS, Jovov B, Berdiev BK, Fuller CM, Dedman JR, Kaetzel M, Benos DJ. Regulation of epithelial sodium channels by the cystic fibrosis transmembrane conductance regulator. J Biol Chem 1996;271:4725-4732.

5. Cheng SH, Rich DP, Marshall J, Gregory RJ, Welsh MJ, Smith AE. Phosphorylation of the R domain by cAMP-dependent protein kinase regulates the CFTR chloride channel. Cell 1991;66:1027-1036.

6. Gregory RJ, Rich DP, Cheng SH, Souza DW, Paul S, Manavalan P, Anderson MP, Welsh MJ, Smith AE. Maturation and function of cystic fibrosis transmembrane conductance regulator variants bearing mutations in putative nucleotide-binding domains 1 and 2. Mol Cell Biol 1991;11:3886-3893.

7. Lukacs GL, Mohamed A, Kartner N, Chang XB, Riordan JR, Grinstein S. Conformational maturation of CFTR but not its mutant counterpart (delta F508) occurs in the endoplasmic reticulum and requires ATP. EMBO J 1994;13:6076-6086.

8. Rowe SM, Accurso F, Clancy JP. Detection of cystic fibrosis transmembrane conductance regulator activity in early-phase clinical trials. Proc Am Thorac Soc 2007;4:387-398.

9. Kerem E, Hirawat S, Armoni S, Yaakov Y, Shoseyov D, Cohen M, Nissim-Rafinia M, Blau H, Rivlin J, Aviram M, et al. Effectiveness of PTC124 treatment of cystic fibrosis caused by nonsense mutations: a prospective phase II trial. Lancet 2008;372:719-727.

10. Lubamba B, Lecourt H, Lebacq J, Lebecque P, De Jonge H, Wallemacq $\mathrm{P}$, Leal T. Preclinical evidence that sildenafil and vardenafil activate chloride transport in cystic fibrosis. Am J Respir Crit Care Med 2008; 177:506-515.

11. Carvalho-Oliveira I, Scholte BJ, Penque D. What have we learned from mouse models for cystic fibrosis? Expert Rev Mol Diagn 2007;7:407417.

12. Cox T, Lachmann R, Hollak C, Aerts J, van Weely S, Herebicek M, Platt F, Butters T, Dwek R, Moyses C, et al. Novel oral treatment of Gaucher's disease with N-butyldeoxynojirimycin (OGT 918) to decrease substrate biosynthesis. Lancet 2000;355:1481-1485.

13. Cox TM, Aerts JM, Andria G, Beck M, Belmatoug N, Bembi B, Chertkoff R, Vom Dahl S, Elstein D, Erikson A, et al.; Advisory Council to the European Working Group on Gaucher Disease. The role of the iminosugar $\mathrm{N}$-butyldeoxynojirimycin (miglustat) in the management of type I (non-neuronopathic) Gaucher disease: a position statement. J Inherit Metab Dis 2003;26:513-526.

14. Elstein D, Hollak C, Aerts JMF, van Weely S, Maas M, Cox TM, Lachmann RH, Hrebicek M, Platt FM, Butters TD, et al. Sustained therapeutic effects of oral miglustat (Zavesca, N-butyldeoxynojirimycin, OGT 918) in type I Gaucher disease. J Inherit Metab Dis 2004;27:757-766.

15. Butters TD, Dwek RA, Platt FM. Imino sugar inhibitors for treating the lysosomal glycosphingolipidoses. Glycobiology 2005;15:43R-52R.

16. Jacob JR, Mansfiels K, You JR, Tennant BC, Kim YH. Natural iminosugar derivatives of 1-deoxynojirimycin inhibit glycosylation of hepatitis viral envelope proteins. J Microbiol 2007;45:431-440.

17. Chapel C, Garcia C, Roingeard P, Zitzmann N, Dubuisson J, Dwek RA, Trépo C, Zoulim F, Durantel D. Antiviral effect of alpha-glucosidase inhibitors on viral morphogenesis and binding properties of hepatitis C virus-like particles. J Gen Virol 2006;87:861-871.

18. Norez C, Noel S, Wilke M, Bijvelds M, Jorna H, Melin P, DeJonge H, Becq F. Rescue of functional delF508-CFTR channels in cystic fibrosis epithelial cells by the alpha-glucosidase inhibitor miglustat. FEBS Lett 2006;580:2081-2086.

19. Antigny F, Norez C, Becq F, Vandebrouck C. Calcium homeostasis is abnormal in cystic fibrosis airway epithelial cells but is normalized after rescue of F508del-CFTR. Cell Calcium 2008;43:175-183.

20. Noël S, Wilke M, Bot AG, De Jonge HR, Becq F. Parallel improvement of sodium and chloride transport defects by miglustat (n-butyldeoxynojyrimicin) in cystic fibrosis epithelial cells. J Pharmacol Exp Ther 2008:325:1016-1023.

21. Dechecchi MC, Nicolis E, Norez C, Bezzerri V, Borgatti M, Mancini I, Rizzotti P, Ribeiro CM, Gambari R, Becq F, et al. Anti-inflammatory effect of miglustat in bronchial epithelial cells. J Cyst Fibros 2008;7: $555-565$.

22. Norez C, Antigny F, Noel S, Vandenbrouck C, Becq F. A CF respiratory cell chronically treated by miglustat acquires a non-CF like phenotype. Am J Respir Cell Mol Biol 2009 Jan 8 [Epub ahead of print].
23. van Doorninck JH, French PJ, Verbeek E, Peters RH, Morreau H, Bijman J, Scholte BJ. A mouse model for the cystic fibrosis delta F508 mutation. EMBO J 1995;14:4403-4411.

24. Nicklas W, Baneux P, Boot R, Decelle T, Deeny AA, Fumanelli M, Illgen-Wilcke B; FELASA (Federation of European Laboratory Animal Science Associations Working Group on Health Monitoring of Rodent and Rabbit Colonies). Recommendations for the health monitoring of rodent and rabbit colonies in breeding and experimental units. Lab Anim 2002;36:20-42.

25. Legssyer R, Huaux F, Lebacq J, Delos M, Marbaix E, Lebecque P, Lison D, Scholte BJ, Wallemacq P, Leal T. Azithromycin reduces spontaneous and induced inflammation in DEltaF508 cystic fibrosis mice. Respir Res 2006;7:134.

26. Minne A, Louahed J, Mahauden S, Baras R, Renauld JC, Vanbever R. The delivery site of a monovalent influenza vaccine within the respiratory tract impacts on the immune response. Immunology 2007;122:316-325.

27. Platt FM, Neises GR, Karlsson GB, Dwek RA, Butters TD. Nbutyldeoxygalactonojirimycin inhibits glycolipid biosynthesis but does not affect N-linked oligosaccharide processing. J Biol Chem 1994;269: 27108-27114.

28. Leal T, Lebacq J, Vanbinst R, Lederman Ch, De Kock M, Wallemacq P. Successful protocol of anaesthesia for measuring transepithelial nasal potential difference in spontaneously breathing mice. Lab Anim 2006; 40:43-52.

29. Knowles MR, Paradiso AM, Boucher RC. In vivo nasal potential difference: techniques and protocols for assessing efficacy of gene transfer in cystic fibrosis. Hum Gene Ther 1995;6:445-455.

30. Middleton PG, Geddes DM, Alton EW. Protocols for in vivo measurement of the ion transport defects in cystic fibrosis nasal epithelium. Eur Respir J 1994;7:2050-2056.

31. Leal T, Fajac I, Wallace HL, Lebecque P, Lebacq J, Hubert D, Dall'Ava J, Dusser D, Ganesan AP, Knoop C, et al. Airway ion transport impacts on disease presentation and severity in cystic fibrosis. Clin Biochem 2008;41:764-772.

32. Colledge WH, Abella BS, Southern KW, Ratcliff R, Jiang C, Cheng SH, MacVinish LJ, Anderson JR, Cuthbert AW, Evans MJ. Generation and characterization of a delta F508 cystic fibrosis mouse model. Nat Genet 1995;10:445-452.

33. Zeiher BG, Eichwald E, Zabner J, Smith JJ, Puga AP, McCray PB Jr, Capecchi MR, Welsh MJ, Thomas KR. A mouse model for the delta F508 allele of cystic fibrosis. J Clin Invest 1995;96:2051-2064.

34. Dalby R, Suman J. Inhalation therapy: technological milestones in asthma treatment. Adv Drug Deliv Rev 2003;55:779-791.

35. Anderson MP, Sheppard DN, Berger HA, Welsh MJ. Chloride channels in the apical membrane of normal and cystic fibrosis airway and intestinal epithelia. Am J Physiol 1992;263:L1-L14.

36. Shcheynikov N, Wang Y, Park M, Ko SB, Dorwart M, Naruse S, Thomas $\mathrm{PJ}$, Muallem S. Coupling modes and stoichiometry of $\mathrm{Cl} / \mathrm{HCO} 3-$ exchange by slc26a3 and slc26a6. J Gen Physiol 2006;127:511-524.

37. Farinha CM, Amaral MD. Most F508del-CFTR is targeted to degradation at an early folding checkpoint and independently of calnexin. Mol Cell Biol 2005;25:5242-5252.

38. Okiyoneda T, Niibori A, Harada K, Kohno T, Michalak M, Duszyk M, Wada I, Ikawa M, Shuto T, Suico MA, Kai H. Role of calnexin in the ER quality control and productive folding of CFTR: differential effect of calnexin knockout on wild-type and DeltaF508 CFTR. Biochim Biophys Acta 2008;1783:1585-1594.

39. Rosser MF, Grove DE, Chen L, Cyr DM. Assembly and misassembly of cystic fibrosis transmembrane conductance regulator: folding defects caused by deletion of F508 occur before and after the calnexindependent association of membrane spanning domain (MSD) 1 and MSD2. Mol Biol Cell 2008;19:4570-4579.

40. Mall M, Grubb BR, Harkema JR, O’Neal WK, Boucher RC. Increased airway epithelial $\mathrm{Na}+$ absorption produces cystic fibrosis-like lung disease in mice. Nat Med 2004;10:487-493.

41. Mall MA, Harkema JR, Trojanek JB, Treis D, Livraghi A, Schubert S, Zhou Z, Kreda SM, Tilley SL, Hudson EJ, et al. Development of chronic bronchitis and emphysema in beta-epithelial $\mathrm{Na}+$ channeloverexpressing mice. Am J Respir Crit Care Med 2008;177:730-742.

42. Guilbault C, De Sanctis JB, Wojewodka G, Saeed Z, Lachance C, Skinner TA, Vilela RM, Kubow S, Lands LC, Hajduch M, et al. Fenretinide corrects newly found ceramide deficiency in cystic fibrosis. Am J Respir Cell Mol Biol 2008;38:47-56. 